\title{
Evaluation of selenium status and effectiveness of selenolin in the treatment of selenium- dependent hepatopathies
}

\author{
Vladimir A. Grin, Eugenia V. Rogaleva, Marina P. Semenenko, Ksenia A. Semenenko, and \\ Elena $V$. Kuzminova \\ Krasnodar Research Center for Animal Husbandry and Veterinary Medicine, Krasnodar, 350055, \\ Russian Federation
}

\begin{abstract}
The article is devoted to the study of the assessment of selenium status in the pathogenesis of liver diseases and the effectiveness of the use of a selenium-containing drug. In the course of studies in animals with clinical signs and high rates of hepatopathies markers - transaminases, in $100 \%$ of cases a low level of selenium was revealed $-0.026 \pm 0.01 \mu \mathrm{g} / \mathrm{ml}$, because of the increased consumption of this microelement on the background of increased free radical oxidation processes in the liver tissue. It was found that intramuscular administration of the drug selenolin at a dose of $20 \mathrm{mg} / 100 \mathrm{~kg}$ four times with an interval of 15 days significantly improved the clinical status of the experimental animals; the effectiveness of therapy was $93.3 \%$. Parenteral administration of selenolin favorably corrected the processes of lipid peroxidation and contributed to a significant $(p<0.01)$ decrease in the level of liver indicator enzymes in comparison with the control by $34.5 \%$ (aspartate aminotransferase) and by $45.8 \%$ (alanine aminotransferase), and also contributed to an increase in the level of selenium to the level of the physiological norm $(0.068 \pm 0.03 \mu \mathrm{g} / \mathrm{ml})$.
\end{abstract}

\section{Introduction}

Intensification of animal husbandry, low adaptive potential of highly productive livestock, violations of the technology of feeding, keeping and exploitation of livestock very quickly lead to a decrease in the level of compensatory-restorative processes of the body, and, as a rule, the implementation of the pathological process in such animals is accompanied by a wide range of homeostatic deviations that take the form of a certain metabolic syndrome because of the functional liver weakness [1-5].

Since hepatopathies have multifactorial nature, they are quite widespread on farms and cause serious economic damage resulting in the decrease in livestock productivity, the birth of weak, often unviable young animals and early retirement of cows from the herd $[1,4]$.

Liver, being the center for maintaining homeostasis, plays an important role in the processes of adaptation and compensation for impaired functions. This is often realized in the form of dysfunction of the antioxidant system, one of the main enzymes of which is glutathione peroxidase, the active center of which includes selenium. The conclusion about the antioxidant function of selenium was first made by the English biochemist A. Diplock in 
1970. The antioxidant role of selenium is mainly associated with the expression of selenoproteins containing selenium as a selenocysteine residue, fully ionized at physiological $\mathrm{pH}$ and acting as an effective redox catalyzer $[6,7]$.

The main biological role of glutathione peroxidase is to detoxify the reactive oxidant, hydrogen peroxide [8-11]. Classical glutathione peroxidase is a protein with a molecular weight of about $85 \mathrm{kDa}$, formed by four identical subunits with a molecular weight of $21 \mathrm{kDa}$, which are organized into square-shaped complexes. Each subunit consists of one selenium atom in the composition of selenocysteine [6, 7, 12-14].

According to the scientific studies of Aboul Soud et al (2011), as a result of selenium deficiency, damage of the cell membranes of hepatocytes is caused by the initiation of lipid peroxidation processes because of the decrease in the activity of the enzyme glutathione peroxidase, while an increase in the concentration of hydroperoxides, malondialdehyde and other lipid peroxidation products leads to the hepatocytes damage and contributes to the progression of the disease $[15,16]$.

Studies have determined the influence of organic forms of selenium on the regulation of antioxidant processes in the liver tissue of rats with experimental toxic hepatitis, and the administration of selenium drugs (sodium selenite, selenophene) promotes a slight acceleration of regeneration processes in liver after its in vivo partial extirpation [12, 16-18].

Thus, on the one hand, the development of hepatopathy leads to a reduced content of selenium in the body, on the other hand, selenium deficiency provokes a decrease in the processes of lipid peroxidation, which is one of the main triggers for the progression of liver diseases, since now there are already numerous data on the role and significance of oxidative stress in the development and progression of a number of pathological processes, primarily in the liver, as an organ most sensitive to the toxic effects of peroxide radicals.

However, the role of selenium status and the degree of its participation in the pathogenesis of the development of hepatopathies as one of the etiological factors in veterinary medicine has been insufficiently studied and requires further research.

The aim of the work was to determine the correlation between selenium deficiency and the occurrence of liver dysfunction, as well as the effectiveness of using the injectable drug selenolin in the treatment of hepatopathies in cows.

\section{Materials and methods}

The studies were carried out on Holstein cows with clinical and biochemical syndrome of hepatopathies. For the experiment, animals were selected with similar physiological and age parameters. The criterion for inclusion in the study was the clinical picture of metabolic disorders of the liver function such as depression, tachycardia, decreased appetite, rare and sluggish chewing, hypotension, dyspeptic manifestations, liver pain on percussion and palpation, increase in the percussion borders of liver, signs of bone tissue demineralization, which was accompanied by softening of the last caudal vertebrae, dullness and fragility of the covering.

To confirm the diagnosis and to clarify the degree of metabolic disorders in the surveyed livestock, blood was taken with its subsequent biochemical study to determine the main markers of liver pathology, indicating an increased load on this organ.

The effectiveness of the use of the injectable drug selenolin in the treatment of hepatopathies was determined in comparison with the positive (analogue drug) and negative control on three groups of cows $(n=30)$ 6-7 months of the dry period, selected taking into account body weight and productivity.

Blood biochemical parameters were studied on an automatic Vitalab Selectra Junior analyzer with software version 1.0. (open system for photometric tests, manufactured by 
Vital Scientific N. V. Netherlands) using reagents from ELITech Clinical Systems (France) and Analyticon biotechnologies AG (Germany).

The correlation dependence between low selenium levels and manifestation of hepatopathy was determined by the level of selenium in the blood of sick animals by the atomic adsorption spectrometry using a Kvant-2 device (Russia).

The influence of selenolin on the processes of peroxidation of unsaturated fatty acids in the blood of cows was assessed by the level of primary products - diene conjugates (DC) and ketodienes (KD) as well as secondary products - malondialdehyde (MDA) and final products - fluorescent Schiff bases. The analysis of the dynamics of indicators of the peroxidation system was carried out in accordance with the methodological recommendations of FSBRI "All-Russian Research Veterinary Institute of Pathology, Pharmacology and Therapy" (2010) for the study of the processes of lipid peroxidation and the antioxidant defense system of the body in animals.

Statistical processing of the obtained results was carried out by the methods of mathematical statistics adopted in biology and medicine [19] using the software of the Mikrosoft ${ }^{\circledR}$ company and the Carl Zeiss ${ }^{\circledR}$ company. The criterion of reliability was determined according to the Student's table, while the average indicator (M) was calculated; to process the research data, descriptive statistics were used, followed by multiple pairwise comparisons using the Newman-Keuls test at a $5 \%$ significance level of differences $(p<0.05)$.

The object of the study is an injectable form of the drug selenolin, which is a $2 \%$ sterile solution of diacetophenonyl selenide (DAFS-25) in vegetable oil. It contains organically bound selenium, which forms, during destruction, selenium-containing fragments similar to natural metabolites of the animal body, which provides a relatively low toxicity and high assimilation of the drug in the animal body.

The animals of the experimental group were injected with selenolin intramuscularly at a dose of $5 \mathrm{ml}(20 \mathrm{mg} / 100 \mathrm{~kg}) 4$ times with an interval of 15 days. The cows of the second experimental group in the same mode of administration received sodium selenite at a dose of $0.2 \mathrm{mg}$ per $1 \mathrm{~kg}$ of animal weight. Animals of the control group were injected with soybean oil in a dose of $5 \mathrm{ml} / 100 \mathrm{~kg}$ according to the similar scheme.

During the experiment, the animals were daily monitored, the severity of the pathological disorder of the main functions of the liver was noted, and the level of antioxidant activity of the body and the main biochemical parameters were studied. Blood samples for biochemical studies were taken at the beginning and at the end of the experiment two hours before morning feeding in accordance with generally accepted methods.

Background studies of blood serum found that the indicators of protein metabolism were characterized by the development of hypoproteinemia in $33.3 \%$ of animals and hyperproteinemia in $66.7 \%$, which was accompanied by an increase in the gamma-globulin fraction and a positive thymol test. The indicators of pigment metabolism testified to the functional weakness of the liver: in $83.3 \%$ of cows, hyperbilirubinemia was determined with the appearance of a conjugated fraction in the blood.

Disorders of lipid-carbohydrate metabolism were characterized by hypocholesterolemia (in $93.3 \%$ ) and hypoglycemia (in 53.3\%), which can be regarded from the point of view of a deficit of metabolic energy in diets and functional disorders of liver.

With regard to the concentration of carotene in the blood, a decrease in this indicator was noted in $100 \%$ of animals because of the insufficient provision of diets and general metabolic disorders.

None of the animals with the detection of symptoms of hepatopathy have an optimal supply of selenium in the blood serum $(0.06-0.12 \mu \mathrm{g} / \mathrm{ml})$. The suboptimal selenium concentration, characterized by the level of micronutrient in the blood serum in the values of $0.06-0.056 \mu \mathrm{g} / \mathrm{ml}$, was recorded only in $3.3 \%$ of cows, in the range of $0.057-0.038 \mu \mathrm{g} / \mathrm{ml}-$ in two cows. In the majority of animals $(90 \%)$, the selenium concentration in the blood serum 
was significantly lower than the reference values, and averaged $0.026 \pm 0.01 \mu \mathrm{g} / \mathrm{ml}$, which can be classified as a deficient state for the microelement. Thus, it was found that in animals with a biochemical profile of hepatopathies, selenium deficiency was detected in $100 \%$ of cases.

The low concentration of selenium in the blood serum of animals can be explained by the depletion of endogenous reserves as a result of the activation of free radical oxidation processes in the liver tissue, for which a microelement is attracted, which is a cofactor of the antioxidant system. According to the literature data, a low level of selenium in the body of animals correlates with the incidence of hepatopathies, i.e., the higher the severity of the disease, the lower the selenium concentration $[4,11,13,16,19-22]$.

At the same time, there is a clear parallel between an increased level of liver pathology markers - transaminases (alanine aminotransferase, aspartate aminotransferase, thymol test) with a decreased level of selenium. In sick animals in comparison with the reference values, dysfermentemia was noted, which in $84 \%$ of cases was caused by an increase in the activity of ALT and AST and in 90\% - ALT and ALP, which can be considered as a differential diagnostic criterion for the violation of the integrity and lesions of hepatocytes, as well as an increase in thymol samples characteristic of chronic mesenchymal inflammatory liver syndrome, accompanied by cholestasis.

On the background of selenium deficiency and an increased level of transaminases in the blood of sick cows, a high status of peroxidation of unsaturated fatty acids (diene conjugates $\left(\mathrm{DC}_{(232)}\right)-0.30 \pm 0.01 \mathrm{AU} / \mathrm{ml}$ of blood, ketodienes $\left(\mathrm{KD}{ }_{(273)}\right)-0.22 \pm 0.02 \mathrm{AU} / \mathrm{ml}$ of blood, secondary products - malondialdehyde $\left(\mathrm{MDA}_{(537)}\right)-1.76 \pm 0.04 \mu \mathrm{Ml} / 1$ and final products - fluorescent Schiff bases (365-370) $-0.22 \pm 0.02 \mathrm{AU} / \mathrm{ml}$ of blood.

\section{Results and discussion}

In a comparative aspect of the conducted studies, it was determined that the therapy with selenolin and sodium selenite improved the clinical condition of the experimental animals. However, it should be noted that the disappearance of the symptoms of hepatopathy in cows of the first experimental group occurred by 18-20 days of treatment, the therapeutic efficiency was $93.3 \%$ (28 animals), while the recovery of animals of the second group was noted only by $23-25$ days, the therapeutic efficiency was at the level of $76.7 \%$ (23 animals). In the control group, the clinical signs of hepatopathy progressed and the disease turned into a recurrent form.

The normalization of the processes of peroxidation of unsaturated fatty acids with the administration of selenolin contributed to the optimization of metabolic processes in the liver by activating the synthesis of selenium-dependent glutathione peroxidase, which was accompanied by the restoration of the protein-synthetic function of liver and the decrease in the level of transaminases (alanine aminotransferase, aspartate aminotransferase) and alkaline phosphate transferase (Table 1).

In the experimental cows, there were positive changes in the biochemical status with the priority of indicators in the first experimental group.

In the experimental groups, the level of total protein returned to normal and was within the physiological norm $(84.1 \pm 6.12-82.7 \pm 5.36 \mathrm{~g} / 1)$. Whereas in the control group, on the background of progression of hepatopathy and increased load on the liver, higher values of total protein were recorded. However, this was due to an increase in the number of animals with hyperproteinemia - 25 heads $(83.3 \%)$.

The restoration of the functional activity of hepatocytes of the hepatic tissue with the administration of selenolin inhibited the development of mesenchymal-inflammatory syndrome (negative thymol test) and contributed to a decrease in the synthesis of the gamma- 
globulin fraction. The level of gamma globulins in the first experimental group was lower than the indicators of cows of the second group and control by $5.1 \%$ and $21.5 \%$, respectively.

Table 1. Influence of selenolin on the biochemical parameters of the blood of cows after intramuscular administration $(\mathrm{M} \pm \mathrm{m} ; \mathrm{n}=30)$

\begin{tabular}{|c|c|c|c|c|}
\hline \multirow[b]{2}{*}{ Indicators } & \multicolumn{4}{|c|}{ Groups of animals } \\
\hline & Background & $\begin{array}{c}1 \text { st } \\
\text { experimental, } \\
\text { selenolin }\end{array}$ & $\begin{array}{c}\text { 2nd } \\
\text { experimental, } \\
\text { sodium } \\
\text { selenite }\end{array}$ & Control \\
\hline Total protein, $\mathrm{g} / \mathrm{l}$ & $78.3 \pm 4.81$ & $84.1 \pm 6.12 *$ & $82.7 \pm 5.36^{*}$ & $93.7 \pm 5.94$ \\
\hline Albumins, \% & $38.8 \pm 5.53$ & $44.6 \pm 7.04$ & $46.3 \pm 6.82$ & $36.4 \pm 5.17$ \\
\hline$\alpha$-globulins, $\%$ & $11.75 \pm 1.88$ & $13.5 \pm 2.13$ & $10.24 \pm 1.62$ & $12.6 \pm 1.59$ \\
\hline$\beta$ - globulins, $\%$ & $7.05 \pm 0.62$ & $6.49 \pm 0.70$ & $6.17 \pm 0.57$ & $5.85 \pm 0.46$ \\
\hline$\gamma$-globulins, $\%$ & $42.4 \pm 6.25$ & $35.4 \pm 4.82$ & $37.3 \pm 5.13$ & $45.1 \pm 6.61$ \\
\hline AST, AU / 1 & $116.2 \pm 7.46$ & $80.8 \pm 6.54 * *$ & $108.5 \pm 6.02$ & $123.3 \pm 8.34$ \\
\hline ALT, AU / 1 & $43.1 \pm 4.18$ & $32.2 \pm 3.79 * *$ & $37.6 \pm 5.41$ & $59.6 \pm 7.22$ \\
\hline $\mathrm{ALP}, \mathrm{AU} / 1$ & $128.4 \pm 9.12$ & $117.2 \pm 12.6$ & $161.4 \pm 8.59$ & $193.2 \pm 11.5$ \\
\hline Thymol test & + & - & - & ++ \\
\hline Glucose, $\mathrm{mmol} / \mathrm{l}$ & $3.1 \pm 0.13$ & $3.7 \pm 0.11$ & $3.3 \pm 0.14$ & $2.9 \pm 0.12$ \\
\hline Cholesterol, $\mathrm{mmol} / \mathrm{l}$ & $3.7 \pm 0.31$ & $4.4 \pm 0.42 * *$ & $3.9 \pm 0.36$ & $3.5 \pm 0.27$ \\
\hline Triglycerides, mmol / 1 & $0.23 \pm 0.01$ & $0.30 \pm 0.02 * *$ & $0.27 \pm 0.02 * *$ & $0.21 \pm 0.01$ \\
\hline Carotene, $\mathrm{mg} \%$ & $0.42 \pm 0.05$ & $0.57 \pm 0.03$ & $0.44 \pm 0.06$ & $0.39 \pm 0.04$ \\
\hline Selenium, $\mu \mathrm{g} / \mathrm{ml}$ & $0.026 \pm 0.02$ & $0.068 \pm 0.03 * *$ & $0.054 \pm 0.02 * *$ & $0.025 \pm 0.01$ \\
\hline $\mathrm{DC}_{(232)}, \mathrm{AU} . / \mathrm{ml}$ of blood & $0.30 \pm 0.01$ & $0.21 \pm 0.03 * *$ & $0.25 \pm 0.02 * *$ & $0.31 \pm 0.03$ \\
\hline $\mathrm{KD}_{(273)}, \mathrm{AU} . / \mathrm{ml}$ of blood & $0.22 \pm 0.02$ & $0.17 \pm 0.01 * *$ & $0.20 \pm 0.02^{* *}$ & $0.23 \pm 0.03$ \\
\hline MDA (537), $\mu \mathrm{Ml} / 1$ & $1.76 \pm 0.04$ & $1.48 \pm 0.03 *$ & $1.57 \pm 0.07 *$ & $1.74 \pm 0.05$ \\
\hline $\begin{array}{l}\text { Schiff bases (365-370), AU / } \mathrm{ml} \text { of } \\
\text { blood }\end{array}$ & $0.22 \pm 0.02$ & $0.19 \pm 0.01 * *$ & $0.20 \pm 0.02 * *$ & $0.23 \pm 0.03$ \\
\hline
\end{tabular}

Note: ${ }^{*}-\mathrm{p} \leq 0.05,{ }^{* *}-\mathrm{p} \leq 0.01,{ }^{* * *}-\mathrm{p} \leq 0.001$

Analysis of the activity of aspartate aminotransferase and alanine aminotransferase in experimental animals showed that the level of these indicator enzymes was significantly ( $p<0.01$ ) lower than the control values by the end of the experimental period, the difference was $34.5 \%$ and $45.8 \%$, respectively.

The introduction of sodium selenite did not allow to have an equally significant effect on the activity of transaminases in the blood of cows of the second experimental group: the concentration of aspartate aminotransferase was at the level of the upper limit values 108.5 $\pm 6.02 \mathrm{AU} / 1$, while the level of alanine aminotransferase at the end of the experiment exceeded the reference values for this indicator and amounted to $37.6 \pm 5.41 \mathrm{AU} / 1$ at a norm of 6.9-35 $\mathrm{AU} / 1$.

Under conditions of increased generation of active oxygen forms in the body, when the self-induced chain mechanism of free radical reactions goes beyond the stationary level, selenolin showed a significant $(\mathrm{p}<0.05 ; \mathrm{p}<0.01)$ inhibitory effect on the synthesis of active oxygen forms in the blood of cows at different levels of their generation.

Therefore, in the dynamics in the experimental group, the decrease in DC was at the level of $30 \%, \mathrm{KD}-22.7 \%$, MDA $-15.9 \%$ and Schiff bases $-13.6 \%$. In a comparative aspect with the indicators of the cows of the second experimental group, the level of lipid peroxidation products was lower by $16.0 \%$ (diene conjugates), $15 \%$ (ketodienes), $5.7 \%$ (malondialdehyde) and $5 \%$ (Schiff bases) in relation to the control values it was lower by $32.3 \%, 26.1 \%, 14.9 \%$ and $17.4 \%$, respectively.

The beneficial effect of selenolin and sodium selenite on the course of metabolic processes in liver was accompanied by the correction of lipid and carbohydrate metabolism. 
The level of glucose and lipids (cholesterol and triglycerides) in both groups at the end of the experimental period had higher values in comparison with control analogs with a tendency of higher values in the first experimental group. In the first experimental group, the excess in terms of glucose in comparison with the control was $27.6 \%$, in the group with an analogue drug it was $13.8 \%$. The level of cholesterol and triglycerides was higher than the indicators of the second experimental group by $12.8 \%$ and $11.1 \%$ and higher than the control indicators by $25.7 \%$ and $42.9 \%$, respectively.

The reparative effect of selenolin on the metabolic functions of liver was also manifested by higher values of carotene $-0.57 \pm 0.03 \mathrm{mg} / \%$, which exceeded the indications of cows that were injected with an analogue drug by $29.5 \%$ and control by $46.2 \%$.

The conducted biochemical studies of blood serum made it possible to reveal the positive dynamics of the selenium level in the experimental animals. Parenteral administration of selenolin contributed to a significant $(\mathrm{p} \leq 0.01)$ increase in selenium levels within the physiological norm $(0.068 \pm 0.03 \mu \mathrm{g} / \mathrm{ml})$ and exceeded the indicators of the second experimental group by $25.93 \%$, and the control indicators in 2.62 times. Higher selenium indices in animals of the first experimental group were due to the fact that selenolin contains selenium in its composition in the form of an organic compound, which has high bioavailability and less toxicity for animals compared to inorganic preparations. According to N.I. Konova (1992), when sodium selenite is used, the absorption of selenium is much lower: in monogastric animals, it is about $74 \%$, and in ruminants only $30 \%$, which is because sodium selenite is rapidly reduced to elemental selenium and forms insoluble forms with other components of mixtures [22].

By the end of the experimental period, the selenium level in the blood of cows from the control group was still at a physiologically low level $-0.025 \pm 0.01 \mu \mathrm{g} / \mathrm{ml}$, which is apparently associated with an increase in pathological processes in liver and an increase in the functional activity of the antioxidant system. These processes are accompanied by an increased use of selenium for the construction of enzymes specific for a given system and lead to a further depletion of the reserves of this element.

The hepatoprotective mechanism of action of selenolin lays in organic selenium, which is part of the drug, as a cofactor, participates in the detoxification of hydrogen peroxide and hydroperoxides of unsaturated fatty acids, thereby inhibiting free radical oxidation processes leading to the formation of extremely reactive hydroxyl radicals, the excessive accumulation of which contributes to the development metabolic syndrome because of the functional liver weakness.

\section{Conclusion}

Thus, intramuscular administration of the drug selenolin into the body of cows helped leveling the manifestation of clinical symptoms of hepatopathy, reducing high levels of hepatic transaminases, thereby ensuring the restoration of metabolic processes in liver. Selenolin in the treatment of hepatopathies weakened the negative effect of xenobiotics and other factors by maintaining the antiradical activity of liver at a sufficient level, activating reduced glutathione and reducing the formation of toxic lipid peroxidation products, thereby providing a pronounced hepatoprotective effect.

\section{References}

1. A. S. Erokhin, Selenium and animal reproductive function, 142 (Moscow, 2008)

2. V. Yu. Kozlovsky, Forest Glades, 274 (2010) 
3. E. V. Rogaleva, V. A. Grin, M. P. Semenenko, Collection of scientific papers of the Krasnodar Research Center for Animal Husbandry and Veterinary Medicine, 9(1), 262 (2020)

4. A. Kalkan, V. Bulut, S. Avci et al, J. Trace Elem. Med. Biol., 16(4), 227 (2002)

5. M. P. Semenenko, D. V. Osepchuk, N. A. Yurina, E. V. Kuzminova, K.A. Semenenko, IOP Conference Series: Earth and Environmental Science, 42048 (2020)

6. H Zeng, Molecules, 14(3), 1263 (2009)

7. V. A. Tutelyan, V. A. Knyazhev, S. A. Khotimchenko et al, Selenium in the human body, 224 (M: Publishing house RAMN, 2002)

8. V.G. Skopichev, L.V. Zhichkina, O.M. Popova et al, Microelementosis of animals: tutorial, 288 (SPb.: Prospectus of Science, 2015)

9. S.T. Kokhan, E.V. Fefelova, M.V. Maksimenya et al., Fundamental research, 11-4, 837 (2012)

10. V.A. Grin, A.A. Abramov, M.P. Semenenko, E.V. Kuzminova, E.V. Rogaleva, E.N. Rud, Veterinary medicine of the Kuban, 2, 6 (2020)

11. S.K. Jain, P.W. Pemberton, A. Smith et al, J. Hepatol., 36(6), 805 (2002)

12. L.L. Zubkova, Kazan science, 3, 241 (2010)

13. G.N. Bliznetsova, PhD thesis abstract, 46 (Voronezh, 2010)

14. A.S. Gasanov, A.A. Gatina, Z.M. Zukhrabova et al, Kazan: Information Technology Center KGAVM, 51 (2020)

15. Yu.M. Stepanov, V.V. Belitsky, S.V. Kosinskaya, Frequent gastroenterology, 3, 65 (2012)

16. M.A. Abou Soud, A.M. Othman, G.E. Desoky et al, J. Toxicol. Sci., 36(3), 285 (2011)

17. A.I. Berdnikov, Scientific potential - modern agro-industrial complex, Izhev State Agr. Acad., 2, 76 (2009)

18. A.V. Boroda, PhD thesis, 101 (Kursk, 2005)

19. A.A. Zverev, T.L. Zefirov, Biometrics ,42 (Kazan: Kazan Federal University, 2013)

20. V.A. Chelnokov, PhD thesis, 169 (Kursk, 2013)

21. T. Himoto, H. Yoneyama, K. Kurokohchi et al., Nutr. Res., 31(11), 829 (2011)

22. A.V. Zholnin, I.A. Myakishev, P.N. Popkov, Bulletin of SUSU, 19, 126 (2010) 